\title{
Capital Social como factor de emprendimiento en los países de la OCDE: implementación de un modelo con datos de panel
}

\author{
MARTíNEZ SiDÓN, GILBERTO \\ Universidad Autónoma de Baja California (Méjico) \\ Correo electrónico: gilberto.mtzs88@gmail.com \\ SAAVEDRA LEYVA, RAFAEL EDUARDO \\ Universidad Autónoma de Baja California (Méjico) \\ Correo electrónico: eduardo.saavedra@uabc.edu.mx \\ MORONES CARRILLO, ANA LOURDES \\ Universidad Autónoma de Baja California (Méjico) \\ Correo electrónico: lourdes_mc39@hotmail.com
}

\begin{abstract}
RESUMEN
En el presente artículo se analiza el capital social como factor de emprendimiento en los países de la OCDE. Se utilizan las bases de datos del Global Entrepreneurship Monitor (GEM) y la World Values Survey (WVS). De la primera se obtiene la variable de emprendimiento y de la segunda se obtienen variables de capital social, como la confianza y la pertenencia a un grupo del tejido social. Se utiliza un modelo de panel con efectos aleatorios. Los resultados sugieren que las variables de capital social impactan positivamente en la actividad de emprendimiento, incrementando su probabilidad de éxito, no solo en la fase inicial sino también en el largo plazo.
\end{abstract}

Palabras clave: emprendimiento, capital social, confianza, empresas.

Clasificación JEL: C13; M13.

MSC2010: 91B38; 91D30; 91G70. 


\title{
Social Capital as an Entrepreneurship Factor in OECD Countries: Implementation of a Model with Panel Data
}

\begin{abstract}
In the present article, social capital is analyzed as entrepreneurship factor across OECD countries. The databases of Global Entrepreneurship Monitor (GEM) and the World Values Survey (WVS) are used. Entrepreneurship variable is obtained from GEM, and social capital variables are obtained from WVS. Confidence and to belong a social group are considerate as social capital variables. A panel model with random effects is used. The results suggest that social capital variables have a positive impact on entrepreneurial activity, increasing their probability of success, not only in the initial phase but also in the long term.
\end{abstract}

Keywords: entrepreneurship, social capital, trust, enterprise.

JEL classification: C13; M13.

MSC2010: 91B38; 91D30; 91G70.

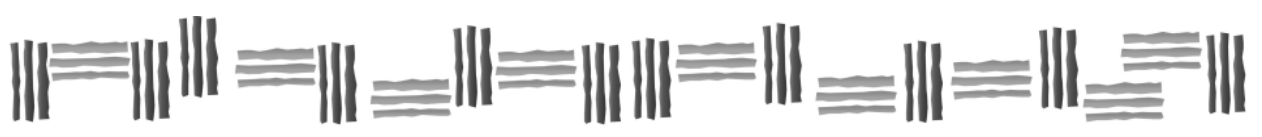




\section{Introducción.}

En la teoría económica se reconoce que el emprendimiento es una de las principales formas de creación de empleo, generación de mayor crecimiento y desarrollo económico, así como un factor clave en la redistribución del ingreso. El emprendimiento se ha analizado desde distintos enfoques. El primero de ellos, se centra en el papel del emprendedor-que destaca las cualidades personales. De acuerdo con esta visión, un emprendedor se caracteriza por la necesidad de logros, el deseo de ser independiente, la tolerancia al riesgo, la perseverancia, la autoconfianza y el aprendizaje constante (Kantis, 2004). En este sentido, diversos autores han definido el concepto de emprendedor dentro del contexto empresarial como aquel que funda una empresa.

Este enfoque ha sido criticado en el sentido de que estas características también pueden observarse en otras personas que no emprenden un negocio, como deportistas, políticos, actores, activistas, entre otros. Lo anterior llevó a redefinir el enfoque, centrándose ahora en el proceso de creación de empresas y no en las cualidades del emprendedor.

De esta forma, se consideró que el proceso de creación de empresas o actividad emprendedora, es un fenómeno vinculado a factores sociales, culturales y económicos (Kantis, 2004). Shapero (1984) considera que la creación de una empresa requiere de cuatro condiciones. La primera es la existencia de factores de desplazamiento, que es lo que lleva a una persona a inclinarse por la decisión de emprender. Pueden ser positivos, como la identificación de una oportunidad o el deseo de realización; o negativos, como la salida del desempleo o la incomodidad con el empleo actual. La segunda condición es la disposición a actuar por parte del emprendedor; la tercera, la credibilidad en el proyecto y, finalmente, la disponibilidad de recursos.

Desde el punto de vista económico, factores como los niveles de beneficio esperados y las barreras de entrada son lo que definen a la actividad emprendedora, por su impacto en la entrada y salida de las empresas a los distintos mercados (Geroski \& Murfin, 1991; Kantis, 2004). Rees y Shah (1986) y Evans y Jovanovic (1989) destacan que variables de capital humano como educación y experiencia laboral incrementan los ingresos esperados, mientras que características personales y aspectos demográficos como la edad o estado civil influyen en la actitud hacia el riesgo.

Otro enfoque que ha ganado especial atención es el que centra su atención en el papel de las redes en el proceso de emprendimiento. De acuerdo con este enfoque, la interacción del emprendedor con otros actores como familiares, amigos, instituciones, empresarios es un aspecto crucial en el proceso de creación de empresas (Johannison, 1996).

Los enfoques mencionados anteriormente no son mutuamente excluyentes y el fenómeno del emprendimiento es complejo, por lo que para tener un análisis más adecuado es necesaria la adopción de un enfoque multidisciplinario.

En lo que respecta al capital social, éste se reconoce como un factor intangible que contribuye a la cooperación y la toma de decisiones en la actividad económica. Si bien es cierto que son múltiples estudios los que abordan los temas anteriores, son pocos los que prueban empíricamente una relación entre éstos. Lo anterior se debe quizás a las múltiples definiciones que se tiene sobre capital social.

El concepto de capital social tomó especial relevancia con los trabajos de Coleman (1988) y Putnam (1995). De sus trabajos se define tal concepto como los aspectos de estructura social que facilitan la toma de decisiones de los agentes dentro de la estructura. Son aspectos de las organizaciones sociales, como las redes, normas y la confianza que permiten la acción y cooperación para el beneficio mutuo.

Sin embargo, el concepto fue criticado por Robert Solow (2000) quien argumenta que el capital implica tres aspectos: 1) extensión en el tiempo, 2) sacrificio deliberado en el presente para el beneficio 
humano y 3) alinealidad. De acuerdo con lo planteado, el capital social no cumple con dichos atributos, en gran medida porque el capital representa un stock productivo o factores naturales de producción.

A pesar de las críticas, y en ocasiones la poca aceptación de este concepto especialmente en la economía, diversos trabajos se han realizado respecto al capital social y su relación con variables económicas. Por ejemplo, Portela y Neyra (2002) relacionan el incremento del Producto Interno Bruto (PIB) con el capital social como variable explicativa para el caso de España. Encuentran una relación positiva y significativa entre el capital social y el PIB. Barrutia y Echebarria (2010) relacionan la investigación, desarrollo e innovación con el capital social para algunas regiones de Italia y España. Encuentran una relación positiva y significativa entre las variables.

Así pues, el capital social se puede asociar con la actividad de emprendimiento. Por ejemplo, Castellano (2005) argumenta que el capital social afecta a las empresas de cuatro formas: 1) dentro de la empresa, promueve una mejor coordinación entre individuos y departamentos, el trabajo en equipo puede mejorar la eficiencia y la calidad, con lo que es probable que trabajadores y accionistas sientan crecer un sentido de orgullo en su trabajo, siendo parte de una entidad que hace una contribución positiva a su comunidad. 2) entre empresas, la confianza es el fundamento de la cooperación, y afrontar negocios en conjunto desarrolla relaciones más profundas que pueden servir en el futuro para otros proyectos. 3) entre sectores, reconocer la importancia de las asociaciones intersectoriales, tanto para negocios sostenibles como para el desarrollo sostenible, promueve sinergias, independencia y capital social entre el sector público, el sector privado y la sociedad civil. 4) dentro de la sociedad, el capital social afecta a los tipos de empresas que son exitosas, creando un ambiente positivo para el desarrollo del sector privado.

Unido a lo anterior, el capital social se puede ligar al emprendimiento de diversas formas. Una de ellas tiene que ver con la oportunidad de reconocer el momento adecuado para emprender, dada la información obtenida a través de la interacción (Ramos et al., 2010). Es aquí donde se pone de manifiesto la necesidad de interactuar con el tejido social, crear vínculos de cooperación, establecer acuerdos y sobre todo generar y transmitir confianza. Pues como en su momento sugirió Fukuyama (1995), solo las sociedades con un alto nivel de confianza social tendrán la capacidad de crear empresas flexibles y de gran escala, necesarias para competir exitosamente en la economía global.

Ante dicho contexto el objetivo del presente documento es explorar el efecto que tiene el capital social como factor explicativo del emprendimiento en los países asociados a la Organización para la Cooperación y Desarrollo Económico (OCDE), que define al capital social como las redes, normas, valores y opiniones compartidas que facilitan dentro y entre grupos una mejor manera en el actuar económico. Dicha organización tiene por objetivo el de promover políticas que mejoren el bienestar económico y social de las personas, y es precisamente el emprendimiento una forma de lograr dicho objetivo. La hipótesis sugerida es que el capital social contribuye de manera positiva al emprendimiento, incrementando la probabilidad de iniciar un negocio, dada la pertenencia de las personas a grupos del tejido social y la confianza existente.

El documento se compone de cinco apartados. En el apartado 2 se presenta la literatura empírica y teórica. En el apartado 3 se muestra la metodología a utilizar y se hace una descripción de los datos; en el apartado 4 se muestran los resultados y en el apartado 5 se concluye.

\section{Literatura asociada al tema.}

Como se señaló en la introducción, los estudios sobre cómo influye el capital social en el emprendimiento son relativamente pocos. Tal como señalan Castillo y Smida (2009), el capital social ha sido ampliamente estudiado en su calidad de factor dinamizador de la construcción de tejido social, y por el contrario la exploración de la naturaleza y aplicaciones de este concepto en el contexto empresarial son menos numerosas. La principal limitación se debe quizás a lo complicado que es la 
cuantificación del capital social, ya que no es un elemento tangible, por lo que no existen elementos claros e identificativos para disponer de una medida exacta. En la literatura se considera a la confianza y la pertenencia a grupos en el tejido social como aproximaciones de ésta (Ramos et al., 2010; Barrutia \& Echebarria, 2010; Portela \& Neira, 2002).

En la misma línea North (1981) y Stiglitz (1999) aproximan el concepto de confianza al de capital social. Se argumenta que la confianza es un requisito previo e importante para el buen funcionamiento de los mercados. Asimismo, se reconoce que ésta reduce los costos de transacción y facilita la cooperación entre los agentes económicos, especialmente en los lugares donde las instituciones, leyes y reglamentos carecen de una creencia básica de honestidad.

De acuerdo con Macauley (1963) la confianza se puede construir con base en la contratación relacional, esto es, mediante la creación de relaciones comerciales con socios o conocidos a través de mecanismos informales. En este sentido, la reciprocidad en las interacciones repetidas permite a las empresas mantener una cooperación. Asimismo, Hornungová (2014) argumenta que la cooperación es esencial en el rendimiento empresarial, y por ello es necesario mantener este factor clave en el desarrollo de las empresas.

Asimismo, Woodruff (2004) sugiere que la confianza se puede construir mediante la reputación, la recopilación de información sobre los nuevos socios comerciales y compartición de información con las redes empresariales. La existencia de redes resulta esencial para el tránsito de información sobre la conducta empresarial.

Strandholm, Marino, Steensma y Weaver (2002) examinaron los efectos de la orientación emprendedora y la cultura nacional sobre la formación de alianzas estratégicas que incluyen acuerdos cooperativos. Los resultados mostraron que a medida que crece la pequeña y mediana empresa se incrementa la necesidad de tener socios confiables y competentes para facilitar el alcance de sus objetivos. Asimismo, Raiser, Rousso, Steves y Teksoz (2007) asocian a la actividad empresarial con el tema de la confianza. Argumentan que para que una empresa pueda subsistir por mucho tiempo, es necesario crear un ambiente de confianza entre sus clientes y proveedores, pues a medida que existe una mayor confianza los servicios se vuelven más eficientes.

Así pues, una de las formas más comunes de cómo se relaciona el capital social con el emprendimiento resulta a partir de cómo una persona puede identificar una oportunidad de abrir un negocio. Sirva de ejemplo lo planteado por Ramos, Medina, Lorenzo y Ruiz (2010), quienes argumentan que la oportunidad de emprender un negocio puede originarse a partir del capital social con el que cuenta el emprendedor. Señalan que el capital social es un factor que ayuda a explicar el emprendimiento desde que se hace posible reconocer una oportunidad, dado que las relaciones del emprendedor con el resto de la sociedad le permiten captar información de mercado que le es útil en la toma de decisión.

En esta misma línea Castillo-Vergara, Álvarez-Marín, Alfaro-Castillo, Sánchez y Pizarro (2018) determinan cuáles son los principales factores de emprendimiento universitario. En primer lugar, identifican un grupo de factores internos y externos y posteriormente aplican el proceso analítico jerárquico para ponderar cada factor. Los resultados muestran a la experiencia laboral y la realización de cursos específicos como los factores internos más importantes para la capacidad emprendedora; mientras que los factores externos más relevantes son el ámbito de desarrollo, las redes y amigos, estos dos últimos relacionados con el capital social.

Gedajlovic, Honig, Moore, Payne y Wright (2013) abogan por la inclusión del capital social dentro de la teoría del emprendimiento, proponiendo un modelo esquemático. En este modelo, el primer esquema a considerar son los antecedentes, en donde los eventos del pasado crean y desarrollan las relaciones y redes. Por consiguiente, el segundo esquema del modelo son las relaciones y las redes, que consiste en un proceso de creación y desarrollo de relaciones y redes que dará origen al capital social. El tercer esquema es el capital social, que dicta la formación de las relaciones y la estructura de las 
redes del presente. Finalmente, el esquema de emprendimiento, donde el capital social será aprovechado individualmente o colectivamente para generar nuevos negocios. Dentro de este último esquema cabe el estudio de Neira et al. (2013) quienes analizan el caso de España, utilizando un modelo de elección discreta tipo logit para determinar la probabilidad de emprender, y que incluye variables de capital social. Los resultados sugieren que las redes sociales son explicativas para el emprendimiento.

Por otra parte, Acs, Audretsch y Lehmann (2013) exponen la teoría de knowledge spillover entrepreneurship, que explica cómo el conocimiento no comercializado se concibe como un área de oportunidad, y que solamente al ser explotado por un emprendedor influirá en la economía y sociedad. La teoría mencionada propone la existencia de un filtro del conocimiento, argumentando que el conocimiento es incapaz de expandirse de manera automática. No obstante, se sugiere que las conexiones sociales evaden el filtro del conocimiento, de tal forma que los individuos puedan aprovechar y emprender utilizando el conocimiento generado (Audretsch \& Keilbach, 2005). A causa de la poca atención que poseen las redes en la teoría de knowledge spillover entrepreneurship, Huggins y Thompson (2015) retoman esta teoría para proponer y enfatizar la introducción del papel de las redes y capital network como fuente de innovación y emprendimiento regional. Por lo tanto, esto incluye que las sociedades con conocimiento, que además presenten capital social, visto como una fluida difusión y confianza entre sus individuos, deberán de presentar mayor actividad emprendedora que se traduciría en mayor crecimiento y bienestar social.

Dentro de los trabajos más recientes sobre el tema de capital social, visto éste desde la perspectiva de la creación de redes, y el emprendimiento, se tiene el de Lamine (2017) donde se aplica la teoría actor-red dentro del proceso emprendedor para comprender la dinámica de las redes empresariales. El autor manifiesta que la teoría actor-red es adecuada para describir la creación de negocios puesto que da seguimiento a las relaciones sociales empresariales, examina profundamente el complejo fenómeno empresarial, contextualiza la situación empresarial, e identifica conexiones sociales y políticas. Asimismo, esta teoría no discrimina entre los factores humanos y no humanos, que tienen un impacto importante en el proceso emprendedor y que a menudo se analizan de forma aislada.

Así pues, la relación que existe entre el emprendedor y el capital social puede brindar una oportunidad a las personas inmigrantes. Bizri (2017) analiza este fenómeno. En su argumento se destaca que una inmigrante puede utilizar el capital social (como las redes) para emprender y prosperar. A través del análisis cualitativo, se identifican cinco elementos relevantes en la puesta en marcha del negocio: actitud unidireccional, percepción del negocio familiar, impulso colectivo, estructura de red distinta y proliferación por tomar oportunidades. A su vez, estos elementos se relacionan con tres dimensiones del capital social: cognitiva, estructural y relacional. Concluye que los emprendedores inmigrantes acuden a una estructura de red local para prestarse apoyo mutuo.

Finalmente, el trabajo de Williams, Huggins y Thompson (2018) examina la relación entre emprendimiento y capital social, conceptualizando este último como un fenómeno de carácter espacial. Los autores analizaron esta relación dentro de comunidades deprimidas, aplicando entrevistas a emprendedores de dichas comunidades. Encontraron que el capital social es un elemento importante en la iniciativa emprendedora de este tipo de comunidades. Adicionalmente se destaca que los emprendedores de estas comunidades ostentan vínculos fuertes con otros individuos de la misma comunidad, pero también presentan débiles relaciones con personas externas a ella, por lo que tienden a generar actividad emprendedora informal.

Cabe señalar que una vez que se generó un emprendimiento a partir del capital social, entendido éste como las redes de cooperación y confianza entre empresas, éstas deben alcanzar un desarrollo óptimo que les permita afrontar los diversos riesgos del mercado. Pues como mencionan Rodríguez et al. (2017) donde las redes y confianza se ha extendido, a tal punto que algunas empresas son totalmente dependientes una de otra, la quiebra de una de ellas desencadenaría un efecto dominó, ocasionando pérdidas económicas en las otras empresas de la comunidad y su salida del mercado si esta situación severa continúa. O bien, en una industria donde las empresas vecinas comparten información y cooperan entre ellas, la debacle de una puede afectar a los colindantes de alrededor. 
En suma, la literatura empírica que relaciona el capital social como factor de emprendimiento es poca, y en cada caso se intenta cuantificar el capital social utilizando desde los conceptos de confianza, cooperación y redes, hasta agrupaciones sociales. En cada caso los resultados fueron favorables a dicha hipótesis de relación directa entre ambas variables.

\section{Metodología y datos.}

Los datos respecto de la variable dependiente como explicativas se presentan a continuación.

- Como variable dependiente se utilizó la TEA (Total Early-Stage Entrepreneurial Activity), que mide el porcentaje de la población de 18-64 años, que es emprendedor naciente o propietariogerente de un nuevo negocio. La base de datos de donde se obtuvo la información fue del Global Entrepreneurship Monitor (GEM).

Para la variable de capital social se proponen dos concepciones. Ambas se obtuvieron de la World Values Survey (WVS). La WVS es una encuesta que comenzó en 1981 a recabar información respecto de valores y creencias humanas en países de Europa. Al día de hoy se aplica en casi 100 países de los diferentes continentes, recabando datos respecto de temas sociales, políticos, económicos, culturales, religiosos, demográficos, entre otros. Asimismo, se reconoce a la WVS como el único estudio académico que recoge información respecto de los temas anteriores de las principales zonas culturales del mundo. La información es de carácter público y gratuito (Para una mayor información respecto de la WVS se puede consultar la página electrónica http://www.worldvaluessurvey.org/wvs.jsp). Las variables propuestas son:

- Cs1: Considera el porcentaje de encuestados que responde que se puede confiar en las personas, al momento de cuestionarle lo siguiente: Generalmente hablando, ¿cree usted que la mayoría de la gente puede ser confiada o hay que tener cuidado con el trato con la gente? Dicha pregunta es utilizada ampliamente para hacer referencia al nivel de confianza existente en la sociedad, que se ajusta en gran medida al concepto de capital social.

- Cs2: Porcentaje de personas que son miembros activos o inactivos de algún tipo de asociación, tales como: iglesia $\mathrm{u}$ otras organizaciones religiosas; asociaciones deportivas o de recreo; organizaciones de arte, música o educativas; sindicatos; partidos políticos; organizaciones medioambientales; asociaciones profesionales; organizaciones de caridad; otras organizaciones voluntarias. Esta variable obedece al capital social que se vincula con la definición de redes sociales.

Asimismo, se utilizan variables de control que de acuerdo con la teoría económica también son explicativas del emprendimiento. Éstas son:

- gdppcp: Producto Interno Bruto (PIB) per cápita, medido en miles de dólares a precios constantes de 2005, en paridad de poder adquisitivo. Obtenido de la base de datos de la OCDE. La variable del PIB ha sido utilizada como explicativa del capital social en algunas investigaciones, sirva de ejemplo el realizado por Portela y Neira (2002). Se espera una relación directa entre las variables.

- dsemp: Desempleo. Porcentaje de personas en edad de trabajar que se encuentran desocupadas. La relación entre las variables puede ser en ambos sentidos. Si el signo del coeficiente resulta positivo y estadísticamente significativo, se estará en una situación donde las personas que no 
tienen empleo tienden a encontrar en el emprendimiento una solución al problema del desempleo. Si el signo del coeficiente resulta negativo y estadísticamente significativo, se estará en una situación donde parte del desempleo se debe al fracaso de un emprendimiento. Esta variable ha sido utilizada como explicativa del emprendimiento en algunos trabajos, por ejemplo, en el de Gawel (2010). Los datos se obtuvieron de la OCDE.

- $\quad t$ _ int: Tasa de interés de largo plazo. Se espera una relación inversa y, por tanto, un coeficiente negativo, es decir, cuanto mayor es la tasa de interés, menor es la posibilidad de que las personas emprendan, ya que a mayor tasa de interés, los créditos son más caros y, por otro lado, el costo de oportunidad de emprender se vuelve mayor si se considera que se puede invertir el dinero. En el análisis realizado por Bawuah et al. (2014), se muestra evidencia empírica entre la relación de esta variable con el emprendimiento. Los datos se obtuvieron de la OCDE.

- inf: Inflación. Medida por el Índice de Precios al Consumidor. Dicha variable representa la estabilidad de la economía. De acuerdo con lo expuesto en la teoría del emprendimiento por oportunidad o necesidad, en época de crisis suele haber despidos y las personas tienden a emprender, mientras que en época de estabilidad económica, el emprendimiento que se realiza suele ser por oportunidad, es decir, con conciencia plena y conocimiento del mercado, porque se visualizó una oportunidad de crecimiento y es más probable que los emprendedores por necesidad salgan del mercado para incorporarse nuevamente al mercado laboral (Galindo et al., 2012). De esta forma, un signo positivo del IPC indicaría que ante un periodo de inflación se produce un incremento de la TEA; por lo que, de acuerdo con la idea anteriormente expuesta, se puede inferir que el número de emprendedores por necesidad fue mayor al número de emprendedores por oportunidad y a la salida de emprendedores del mercado si las hubiera. De manera opuesta, un signo negativo del IPC indicaría que ante un periodo de reducción de inflación (o estabilidad) se produce un aumento de la TEA; con lo que se puede inferir que la cantidad de emprendimientos por oportunidad es mayor a la cantidad de emprendimientos por necesidad y a la salida de emprendedores por necesidad del mercado. Por lo tanto, en este caso, el sentido del impacto de esta variable indicaría el tipo de emprendimientos que prevalece. La información se obtuvo de la base de datos de Banco Mundial.

Los países de la OCDE seleccionados fueron aquellos de los que se tiene información tanto de la GEM como de la WVS. La muestra se acotó a 19 países: Australia, Canadá, Estonia, Francia, Alemania, Hungría, Italia, Japón, Corea del sur, México, Holanda, Nueva Zelanda, Noruega, Polonia, Eslovenia, España, Suecia, Turquía y Estados Unidos.

Los años considerados son cuatro, 2001, 2004, 2009 y 2014 (se comienza en el año 2001 dado que GEM empezó a reportar datos a partir de esa fecha. Además, para este año se tomó como referencia el periodo 1994-1998 para aproximar los valores de las variables de capital social), mismos que contemplan los últimos cuatro periodos en que se aplicó la WVS, estos son, 1994-1998, 1999-2004, 2005-2009, 2010-2014 (cabe señalar que la encuesta para el periodo 2015-2018 aún no cuenta con el total de datos recabados de los países donde se aplica). Lo anterior da un total de 76 observaciones.

Dado que la información combina series de tiempo y de corte transversal, se optó por aplicar un modelo con datos de panel. La estructura general del modelo de panel se puede expresar de la siguiente manera:

$$
Y_{i t}=\propto_{i t}+\beta x_{i, t}+u_{i, t}, \quad u_{i, t} \sim N\left(0, \sigma^{2}\right)
$$


donde:

$Y=$ Variable dependiente.

$\propto=$ Intercepto

$\beta=$ Coeficientes a estimar

$x=$ Vector de variables explicativas

$u=$ Término de error

$i=$ Número de individuos

$t=$ Numero de periodos

Un modelo de panel puede variar dependiendo de la estructura de los datos, esto es:

- Modelo agrupado. Se presenta cuando la estructura de datos tiene el mismo intercepto para todos los individuos, esto es: $Y_{i t}=\propto+\beta x_{i, t}+u_{i, t}, \quad u_{i, t} \sim N\left(0, \sigma^{2}\right)$

- Modelo de panel con efectos fijos. Varía dependiendo del intercepto, esto es:

- Efectos fijos individuales.

$$
Y_{i t}=\alpha_{i}+\beta x_{i, t}+u_{i, t}, \quad u_{i, t} \sim N\left(0, \sigma^{2}\right)
$$

- Efectos fijos temporales

$$
Y_{i t}=\propto_{t}+\beta x_{i, t}+u_{i, t}, \quad u_{i, t} \sim N\left(0, \sigma^{2}\right)
$$

○ Efectos fijos individuales y temporales:

$$
Y_{i t}=\propto_{i t}+\beta x_{i, t}+u_{i, t}, \quad u_{i, t} \sim N\left(0, \sigma^{2}\right)
$$

- Modelo de panel con efectos aleatorios. Se presenta cuando el intercepto es totalmente aleatorio, es decir, se descompone en su parte estocástica correspondiente al individuo $\left(\varepsilon_{i}\right)$ y su parte constante $(\propto)$, esto es:

$$
\circ \propto_{i t}=\propto+\varepsilon_{i,} \quad \quad \varepsilon_{i,} \sim N\left(0, \sigma^{2}\right)
$$

Lo que da paso a la siguiente estructura de panel:

$$
\begin{gathered}
Y_{i t}=\alpha+\beta x_{i, t}+v_{i, t}, \quad v_{i, t} \sim N\left(0, \sigma^{2}\right) \\
\text { donde: } \quad v_{i, t}=u_{i, t}+\varepsilon_{i}
\end{gathered}
$$

Expuesto lo anterior, para determinar la especificación adecuada del modelo se recurrió a distintas pruebas. En primer lugar, se calculó el estadístico F, que evalúa si el modelo corresponde a una especificación de efectos fijos como en la ecuación 3.1, o si el modelo corresponde a datos agrupados como en la ecuación 2, en cuyo caso su estimación sería con Mínimos Cuadrados Ordinarios (MCO). La prueba se especifica como:

$$
F_{N-1, N T-K-N}=\frac{\left(R_{n r}^{2}-R_{r}^{2}\right) /(N-1)}{\left(1-R_{u}^{2}\right) /(N T-N-K)}
$$

donde $R_{n r}^{2}$ indica el coeficiente de determinación del modelo general de la forma no restringida (ecuación 3.1), $R_{r}^{2}$ indica el coeficiente de determinación del modelo restringido (ecuación 2). "N" indica el número de observaciones (individuos), "T" el número de periodos, y "K" el total de variables 
explicativas. La hipótesis nula (H0) de la prueba es la existencia de intercepto común $\left(\propto_{1}=\propto_{2}=\right.$ $\left.\propto_{3} \ldots=\propto_{n-1}\right)$. Si se acepta la H0, entonces la estructura de panel no aporta información relevante, por lo que estimar el modelo de panel con MCO resulta adecuado.

Si la hipótesis es rechazada, entonces se debe realizar la prueba de Breusch y Pagan (1980), en cuyo caso se evalúa si el modelo corresponde a una especificación de efectos aleatorios (ecuación 4.2) o de datos agrupados (ecuación 2). El estadístico de prueba se presenta a continuación:

$$
L M=\frac{N T}{2(T-1)}\left[\frac{\sum_{i=1}^{N}\left(\sum_{t=1}^{T} e_{i, t}\right)^{2}}{\sum_{i=1}^{N} \Sigma_{t=1}^{T} e_{i, t}^{2}}\right]^{2}
$$

donde $e_{i, t}$ corresponde a los residuos de la estimación con MCO de la ecuación 2. La H0 indica que la varianza del error no cambia, esto es: $\operatorname{Var}\left(\mathrm{u}_{\mathrm{i}}\right)=0$. Por lo tanto, de aceptarse la H0, la estructura de panel de efectos aleatorios no aporta información.

Si se rechaza la $\mathrm{H} 0$ en ambas, o una de las pruebas anteriores, se recurre a la prueba de Hausman (1978), que ayuda a evaluar si la mejor especificación del modelo es con efectos aleatorios o fijos. El estadístico de prueba es:

$$
H=\left(\widehat{\beta_{f}}-\widehat{\beta_{a}}\right)^{\prime}\left(\widehat{\sigma_{\beta_{f}}^{2}}-\widehat{\sigma_{\beta_{a}}^{2}}\right)^{-1}\left(\widehat{\beta_{f}}-\widehat{\beta_{a}}\right)
$$

donde $\widehat{\beta_{f}}$ corresponde a los estimadores del modelo de efectos fijos, y $\widehat{\beta_{a}}$ corresponde a los estimadores del modelo con efectos aleatorios calculados por Mínimos Cuadrados Generalizados (MCG). La H0 del estadístico indica que los estimadores de MCG son consistentes, pues $\left[\operatorname{Cov}\left(\mathrm{X}_{\mathrm{i}, \mathrm{t}}, \mathrm{u}_{\mathrm{it}}\right)\right]=0$. Si la $\mathrm{H} 0$ no se rechaza, entonces la mejor especificación es un modelo de panel con efectos aleatorios. Cabe señalar que es muy común no rechazar la H0 cuando la temporalidad de los datos es muy corta.

\section{Resultados.}

Los resultados del modelo se presentan en la Tabla 1. De acuerdo con las pruebas de especificación, la mejor estructura es la que contempla efectos aleatorios, esto es:

$$
\begin{gathered}
T E A_{i t}=\propto+\beta_{1} C s 1_{i, t}+\beta_{2} C s 2_{i, t}+\beta_{3} g d p p c p_{i, t}+\beta_{4} d s e m p_{i, t}+\beta_{5} t_{-} i n t_{i, t}+\beta_{4} i n f_{i, t}+v_{i, t} \\
\text { donde: } v_{i, t}=u_{i, t}+\varepsilon_{i}, \quad v_{i, t} \sim N\left(0, \sigma^{2}\right) ; \quad i=\text { país, } t=a \tilde{n} o
\end{gathered}
$$

De acuerdo con los resultados, la inflación $\left(\right.$ inf $_{\mathrm{i}, \mathrm{t}}$ ) tiene un signo positivo, lo que sería indicativo de que los movimientos de la TEA ante la inflación son mayoritariamente influenciados por el emprendimiento por necesidad; sin embargo, la variable no resultó estadísticamente significativa. El resto de variables resultaron con el signo esperado y estadísticamente significativas.

Las variables de capital social que se utilizaron en el modelo resultaron estadísticamente significativas. La variable de capital social que hace referencia a la confianza $\left(\operatorname{cs} 1_{i, t}\right)$ indica que ante un incremento en un punto porcentual en la confianza en otras personas el emprendimiento se incrementa en 0.084 puntos porcentuales. Por su parte, la variable de capital social referente a los grupos sociales o redes $\left(c s 2_{i, t}\right)$ indica que ante un incremento en un punto porcentual en la pertenencia a un grupo social el emprendimiento se incrementa en 0.911 por ciento. Con lo anterior se demuestra que el capital social, visto como nivel de confianza y pertenencia a grupos sociales, potencializa la capacidad de emprender en mayor medida que el resto de las variables explicativas del emprendimiento. 
La variable de desempleo $\left(\operatorname{dsemp}_{i, t}\right)$ resultó con un coeficiente positivo y estadísticamente significativo. Lo que implica que abrir una empresa es una de las principales opciones para combatir la problemática de la falta de empleo. Ante un incremento de un punto porcentual en el nivel de desempleo, el emprendimiento se incrementa en 0.3895 puntos porcentuales.

Tabla 1. Resultados del modelo.

\begin{tabular}{|c|c|c|}
\hline \multicolumn{3}{|c|}{ Variable Endógena: $T E A_{i, t}$} \\
\hline \multirow[t]{3}{*}{ Variables Exógenas } & \multicolumn{2}{|c|}{ Coeficientes } \\
\hline & 1.1078 & * \\
\hline & $(0.5405)$ & \\
\hline \multirow{2}{*}{$\operatorname{cs} 1_{i, t}$} & 0.0843 & $* *$ \\
\hline & $(0.0335)$ & \\
\hline \multirow{2}{*}{$\operatorname{cs} 2_{i, t}$} & 0.9112 & $* * *$ \\
\hline & $(0.2205)$ & \\
\hline \multirow{2}{*}{$d s e m p_{i, t}$} & 0.3895 & $* *$ \\
\hline & $(0.1201)$ & \\
\hline \multirow{2}{*}{$t_{-} i n t_{i, t}$} & -0.0177 & $* *$ \\
\hline & $(0.0021)$ & \\
\hline \multirow{2}{*}{$\inf f_{i, t}$} & 0.0024 & \\
\hline & $(0.0016)$ & \\
\hline \multirow{2}{*}{$g d p p c p_{i, t}$} & -0.0988 & $*$ \\
\hline & $(0.0494)$ & \\
\hline Núm. Observaciones & & \\
\hline R-2 ajustado & 0.8 & \\
\hline D.T. de la variable dependiente. & 0.0 & \\
\hline D.T. de la regresión & 0.0 & \\
\hline Valor p (del estadístico F) & 0.0 & \\
\hline
\end{tabular}

Notas: un asterisco $*$, dos asteriscos $* *$ y tres asteriscos $* * *$ denotan la significancia al 10, $5 \mathrm{y}$ $1 \%$ respectivamente. El valor en el paréntesis denota el error estándar.

\section{Contraste $\mathbf{F}$ (diferentes interceptos por grupos):}

$\mathrm{H} 0=$ los grupos tienen un intercepto común.

Valor $\mathrm{p}$ de Estadístico $\mathrm{F}=0.0027$

Contraste de Breusch-Pagan (efectos grupales):

$\mathrm{H} 0=$ Varianza del error igual a cero, $\operatorname{Var}\left(u_{i}\right)=0$

Valor p de Estadístico Chi cuadrado $X^{2}=0.0000$

Contraste de Hausman (efectos aleatorios vs efectos fijos):

$\mathrm{H} 0($ efectos aleatorios $)=$ Estimadores de MCG son consistentes, $\left[\operatorname{Cov}\left(X_{i, t}, u_{i t}\right)\right]=0$

Valor $\mathrm{p}$ de Estadístico Chi cuadrado $X^{2}=0.8990$

Fuente: Elaboración propia.

En cuanto a la variable de ingreso per cápita, que en la literatura se asocia como una medida de desarrollo, ésta resultó significativa y con signo negativo. Lo anterior coincide con lo propuesto por Schultz (1991), quien señala que existe una relación negativa entre el nivel de desarrollo económico y la evolución del factor emprendedor. Kuznets (1971) se apoya en las consecuencias del cambio tecnológico, para justificar el desplazamiento de la actividad económica desde los sectores estancados o en declive hacia otros en crecimiento y una relación negativa entre el empleo por cuenta propia y la tasa de desarrollo.

Asimismo, el coeficiente de la tasa de interés cumple con lo esperado, dado que un aumento de un punto porcentual en dicha variable disminuirá la actividad emprendedora en 0.0177 puntos porcentuales. Por lo tanto, la tasa de interés incrementa los costos de oportunidad de emprender, dificultando la obtención de financiación e impidiendo la generación de nuevos negocios. 


\section{Conclusiones.}

El factor del emprendimiento es de especial importancia en la actividad económica por ser una fuente empleo y una manera de generar mejores condiciones y bienestar económico. En este sentido, la OCDE ha reconocido esta actividad como una estrategia para alcanzar mejores niveles de desarrollo económico. Por otro lado, se ha reconocido que el capital social puede potenciar muchas actividades y reducir los costos asociados. Por ejemplo, en el caso de la toma de decisiones colectivas, el compartir información, la difusión de innovaciones y, por supuesto, el emprendimiento.

De acuerdo con los resultados obtenidos, se sugiere una relación positiva entre el nivel de emprendimiento y el capital social. Las variables propuestas para medir el capital social a través de la confianza y la pertenencia a un grupo del tejido social, resultaron buenos parámetros. En este sentido, es importante que se puedan formar relaciones confiables y duraderas entre los distintos grupos sociales, de manera que esto influya positivamente en el emprendimiento, y éste a su vez, en el crecimiento y posteriormente en el desarrollo económico.

Con las variables explicativas de control como el PIB per cápita, se comprueba la relación inversa entre el grado de emprendimiento y el desarrollo económico. Esto dada la relación directa obtenida entre el nivel de desempleo y el emprendimiento. Por lo anterior, es importante poner especial atención y focalizar políticas de apoyo a la apertura de empresas, pues éstas surgen como una muy buena alternativa a la generación de empleo durante las épocas de recesión y crisis económica.

Asimismo, es importante poner atención en las tasas de interés, pues tasas de interés altas hacen más difícil la actividad de emprendimiento al encarecer la inversión. Dirigir políticas gubernamentales de apoyo al emprendedor con tasas de interés preferenciales, es deseable como apoyo al emprendimiento.

Finalmente, y a manera de retomar el efecto del capital social sobre el emprendimiento, se hace necesaria una mayor participación de los agentes económicos con el tejido social, pues esto se traduciría en beneficios para toda la red, debido al incremento del conocimiento sobre el entorno, las fortalezas, debilidades, así como oportunidades y amenazas, de tal forma que se encuentren nichos y oportunidades de mercado en las que incursionar con mayor probabilidad de éxito y asegurar la permanencia en el largo plazo, pues es muy común que las empresas no logren sobrevivir por mucho tiempo.

\section{Referencias}

Acs, Z., Audretsch D., \& Lehmann E. (2013). The Knowledge Spillover Theory of Entrepreneurship. Small Business Economics, 41(4), 757-774.

Audretsch, D., \& Keilbach M. (2005). Entrepreneurship Capital and Regional Growth. The Annals of Regional Science, 39(3), 457-469.

Barrutia, J.M., \& Echebarria C. (2010). Social Capital, Research and Development, and Innovation: An Empirical Analysis of Spanish and Italian Regions. European Urban and Regional Studies, 17(4), 371-385.

Bawuah, B., Sare Y., \& Musah A. (2014). The effect of Interest Rate on Micro, Small and Medium Enterprises Financing Decision in Wa Municipality of Ghana. International Journal of Business, Humanities and Technology, 4(4), 81-90.

Bizri, R. (2017). Refugee-Entrepreneurship: a Social Capital Perspective. Entrepreneurship and Regional Development, 29, 847-868. 
Breusch, T.S., \& Pagan A.R. (1980). The Lagrange multiplier test and its applications to model specification in econometrics. Review of Economic Studies, 47, 239-253.

Castellano, H. (2005). La planificación del desarrollo sostenible: Contenidos, entorno y método. Caracas: Cendes.

Castillo, R., \& Smida, A. (2009). El rol del capital social del emprendedor en la expansión exitosa de una Start-up. El caso de hipertexto LTDA. La librería de la U. Estudios Gerenciales, 25(113), 1536.

Castillo-Vergara, M., Álvarez-Marín A., Alfaro-Castillo M., Sánchez, J., \& Pizarro I. (2018). Factores Clave en el Desarrollo de la Capacidad Emprendedora de Estudiantes Universitarios. Revista de Métodos Cuantitativos para la Economía y la Empresa, 25, 111-129.

Coleman, J.S. (1988). Social capital in the creation of human capital. American Journal of Sociology, 94, 95-120. DOI: $10.1086 / 228943$.

Evans, D., \& Jovanovic, B. (2018). An Estimated Model of Entrepreneurial Choice Under Liquidity Constraints. Journal of Political Economy, 97(4), 808-827.

Fukuyama, F. (1995). Trust: The Social Virtues and the Creation of Prosperity. New York: Free Press.

Galindo, M., \& Méndez T. (2012). Innovación y crecimiento económico: Factores que Estimulan la innovación, Cuadernos de Gestión, 12, 51-58.

Gawel, A. (2010). The Relationship between Entrepreneurship and Unemployment in the Business Cycle. Journal of international Study, 4(1), 59-69. DOI: 10.14254/2071-8330.2010/3-1/7.

Gedajlovic, E., Honig B., Moore, C., Payne, G., \& Wright, M. (2013). Social Capital and Entrepreneurship: a Schema and Research Agenda. Entrepreneurship Theory and Practice, 37(3), 455-478.

Geroski, P.A., \& Murfin, A. (1991). Entry and Intra-Industry Mobility in the UK Car Market. Oxford Bulleting of Economics and Statistics, 53(4), 341-359.

Hausman, J.A. (1978). Specification tests in econometrics. Econometrica, 46(6), 1251-1271.

Hornungová, J. (2014). Factor Analysis: An Instrument for Selection of Performance Factors. Revista Métodos Cuantitativos para la Economía y la Empresa, 17, 121-136.

Huggins, R., \& Thompson P. (2015). Entrepreneurship, Innovation and Regional Growth: a Network Theory. Small Business Economics, 45(1), 103-128.

Johanninson, B. (1996). The Dynamic of Entrepreneurial Networks. En P.S. Reynolds (ed.), Frontiers of Entrepreneurship (pp. 253-267), Babson College.

Kantis, H. (2004). Un enfoque sistémico de la creación de empresas. En H. Kantis, P. Angelelli, V. Moori Koenig (Eds.), Desarrollo Emprendedor: América Latina y la experiencia Internacional, (pp. 21-34). Banco Interamericano de Desarrollo. Fundes Internacional.

Knack, S., \& Keefer, P. (1997). Does Social Capital Have an Economic Payoff? A Cross-Country Investigation. Quarterly Journal of Economics, 112(4), 1251-1288. DOI: 10.1162/003355300555475. 
Kuznets, S. (1971). Economic growth of nations, total output and production structure. Massachusetts: Harvard University Press.

Lamine, W. (2017). The Social Network and Entrepreneurial Process: A Sociotechnical Approach. Thunderbird International Business Review, 59(5), 623-633.

Macauley, S. (1963). Non-Contractual relationship in business: A preliminary study. American Sociological Review, 28(1), 55-70.

Neira, I., Portela, M., Cancelo, M., \& Calvo, N. (2013). Social and human capital as determining factors of entrepreneurship in the Spanish Region. Investigaciones Regionales, 26, 115-139.

North, D. (1981). Structure and Change in Economic History. New York: Norton.

Portela M., \& Neira I. (2002). Capital social: concepto y estudio econométrico sobre capital social en España. Estudios Económicos de Desarrollo internacional, 2(2), 25-52.

Putnam, R. (1995). Bowling alone: American's declining social capital. Journal of Democracy, 6(1), 65-78. DOI: $10.1353 /$ jod.1995.0002.

Raiser, M., Rousso, A., Steves F., \& Teksoz, U. (2007). Trust in Transition: Cross-country and firm evidence. The Journal of Law, Economics, and Organization, 24(2), 407-433. DOI: 10.1093/jleo/ewm060.

Ramos, A.R., Medina, J.A., Lorenzo, J.D., \& Ruiz, J. (2010). What you know or who you know? The role of intellectual and social capital in opportunity recognition. International Small Business Journal, 18(6), 566-582.

Rees, H., \& Shah, A. (1986). An Empirical Analysis of Self-Employment in the U.K. Journal of Applied Econometrics, 1(1), 95-108.

Rodríguez, C., Maté, M., \& López, F. (2017). El Contagio en el Fracaso Empresarial como Consecuencia de la Proximidad Geográfica: un Análisis con los Estadísticos Join-Count Aplicado al Sector Servicios. Revista Métodos Cuantitativos para la Economía y la Empresa, 23, 75-97.

Schultz, D.E. (1991). Integrated marketing communications: the status of integrated marketing communications programs in the US today. Journal of Promoting Management, 1(1), 98-104.

Shapero, A. (1984). The Entrepreneurial Event. En C. A. Kent (Ed), The environment for Entrepreneurship, (pp. 21-40). Toronto, Canada: Lexington Books.

Solow, R. (2000). Notes on social capital and economic performance. En, Dasgupta P., \& Sarageldin, Social capital: A multifaceted perspective, World Bank, pp. 6-10.

Stiglitz, J. (1999). Wither Reform? Ten Years of Transition. Keynote Adress, World Bank, Annual Conference on Development Economics, pp. 32.

Strandholm, K., Marino, L., Steensma, H.K., \& Weaver, K.M. (2002). Harnessing Complexity: The Moderating Effect of National Culture on the Relationship Between Entrepreneurial Orientation and Strategic Alliance Portfolio Complexity. Journal of Entrepreneurship: Theory and Practice, 26, $145-161$. 
Williams, N., Huggins, R. \& Thompson, P. (2018). Entrepreneurship and Social Capital: Examining the Association in Deprived Urban Neighborhoods, International Journal of Urban and Regional Research, 44(2), 289-309. DOI: 10.1111/1468-2427.12589.

Woodruff, C. (2004). Establishing confidence in business partners: courts, networks and relationship and pillars of support. En J. Kornai, B. Rothstein, S. Rose-Ackerman (eds.), Creating Social Trust in Post-Socialist Transition (111-125). New York: Palgrave Macmillan. DOI: 10.1057/9781403980663_7. 